\title{
Precise Correction of Lateral Chromatic Aberration in Images
}

\author{
Victoria Rudakova and Pascal Monasse \\ Université Paris-Est, LIGM (UMR CNRS 8049), \\ Center for Visual Computing, ENPC, F-77455 Marne-la-Vallée \\ \{rudakovv, monasse\}@imagine.enpc.fr
}

\begin{abstract}
This paper addresses the problem of lateral chromatic aberration correction in images through color planes warping. We aim at high precision (largely sub-pixel) realignment of color channels. This is achieved thanks to two ingredients: high precision keypoint detection, which in our case are disk centers, and more general correction model than what is commonly used in the literature, radial polynomial. Our setup is quite easy to implement, requiring a pattern of black disks on white paper and a single snapshot. We measure the errors in terms of geometry and of color and compare our method to three different software programs. Quantitative results on real images show that our method allows alignment of average 0.05 pixel of color channels and a residual color error divided by a factor 3 to 6 .
\end{abstract}

Keywords: chromatic aberration, image warping, calibration, polynomial model, image enhancement.

\section{Introduction}

In all optical systems refraction causes the color channels to focus slightly differently. This phenomenon is called chromatic aberration (hereafter CA). When the digital image is obtained, the color channels are misaligned with respect to each other and therefore the phenomenon manifests itself as colored fringes at image edges and high contrast areas. With the increase of sensor resolution for many consumer and scientific cameras, the chromatic aberration gets amplified. For high precision applications, when usage of color information becomes important, it is necessary to accurately correct such defects. Figure 1 shows the effect of our CA correction on real image. CA is classified into two types: axial and lateral. The former occurs when different wavelengths focus at different distances from the lens - in digital images it produces blurring effect since blue and red channels are defocused (assuming the green channel is in focus). A lateral defect occurs when the wavelengths focus at different points on the focal plane and thus geometrical color plane misalignments occur.

In order to define a magnitude of high precision correction, a visual perception experiment was done for different misalignment levels (in pixel units). A synthetic disk was generated on a large image with misalignment between channels

R. Klette, M. Rivera, and S. Satoh (Eds.): PSIVT 2013, LNCS 8333, pp. 12-22, 2014.

(C) Springer-Verlag Berlin Heidelberg 2014 

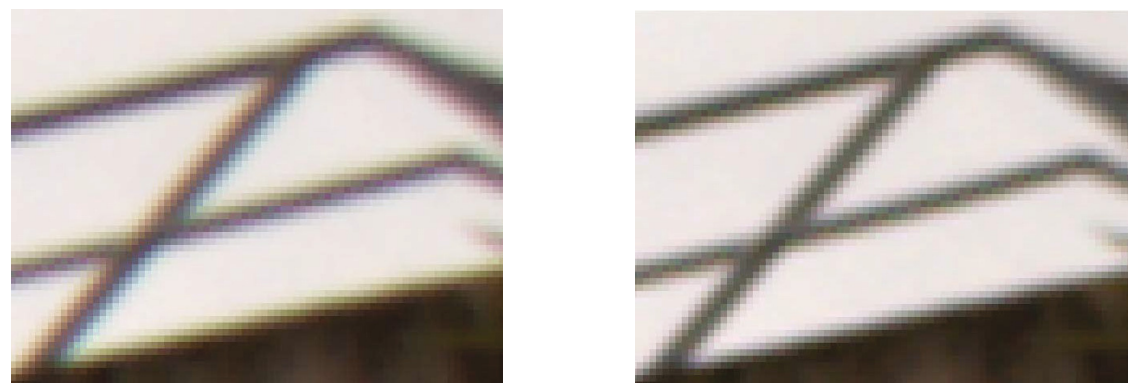

Fig. 1. Cropped and zoomed-in image from camera Canon EOS 40D, before (left) and after (right) chromatic aberration correction by our method. Notice the attenuated color fringes at edges between left and right images.

introduced, then the image was blurred in order to avoid aliasing, and downsampled. A part of the downsampled image was cropped and zoomed-in in Figure 2 It can be noted that 0.1 pixel misalignment is a borderline when aberration becomes just-noticeable, while misalignments of 0.3 pixel and higher are quite perceptible.

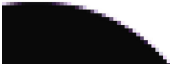

(a) $d=0.05$

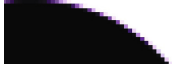

(b) $d=0.1$

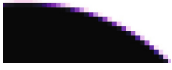

(c) $d=0.2$

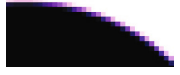

(d) $d=0.3$

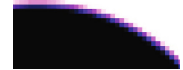

(e) $d=0.5$

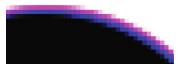

(f) $d=1$

Fig. 2. Visual perception tests for chromatic aberration on synthetic disk image for different values of displacements $d$ (in pixels). Note that a displacement value of 0.1 pixel is just noticeable, while 0.3 pixel displacement is quite perceptible.

Rare papers propose to compensate both axial and lateral CA. The optical solutions 12] try to overcome this effect, but they can be quite expensive and not always effective for the zones farther from the optical center. Another example [3] proposes an active lens system based on modification of the camera settings (magnification, focus, sensor shifting) for each color channel. Such approach may not be practical since it requires taking three pictures for each channel under different camera settings. Kozubek and Matula [4] show how to correct for both types of aberrations in the environment of fluorescent microscopy; however, this method cannot be applied for camera imaging systems.

As most other approaches, we address the lateral CA only but require a single image. Like Boult and Wolberg [5], we formulate the correction as an image warping problem, which means re-aligning color channels digitally. However, Bould and Wolberg [5] do not use any aberration model, interpolating the correction of control points, whereas all other warping methods assume radial nature of the distortion. Matsuoka et al. [6] provide an evaluation of the state of art correction methods, all of which use different ways to model the radial distortion [789]. 
However, it is important to note that not all cameras can be satisfactorily corrected by the same radial model. We will see that more accurate results could be achieved with a more general bivariate polynomial model.

Our lateral CA correction is achieved through realigning the red and blue color planes to the reference green plane. Accurate keypoints (which are centers of disks from a circle pattern) are extracted in each channel independently as described by Section 2. The geometric displacements between color planes is then modelled by bivariate polynomials in Section 3 . Section 4 exposes the quantitative results in terms of both color and geometry misalignments, compares to other software and provides real scene examples. We draw some conclusions in Section 5

\section{Keypoint Detection}

The calibration pattern is made of disks whose centers are used as keypoints. A disk is a filled black circle of fixed radius. High precision requires accounting for a tilt of the camera with respect to the pattern plane normal, thus the disks are viewed as slightly elliptic shapes. We are thus interested in precise ellipse center detection, which is obtained by an adjustment of a parametric model simulating the CCD response using an iterative optimization process. The parametric model takes into account both geometry and intensity.

The geometric model, describing the relationship between model point $(x, y)$ of the filled circle and image point $\left(x^{\prime}, y^{\prime}\right)$, is represented by a local affine transform:

$$
\left(\begin{array}{l}
x \\
y \\
1
\end{array}\right)=\left(\begin{array}{ccc}
\lambda_{1} \cos \theta & -\lambda_{2} \sin \theta & 0 \\
\lambda_{1} \sin \theta & \lambda_{2} \cos \theta & 0 \\
0 & 0 & 1
\end{array}\right) \cdot\left(\begin{array}{c}
x^{\prime}-t_{u} \\
y^{\prime}-t_{v} \\
1
\end{array}\right),
$$

depending on the five geometry parameters, $\left(t_{u}, t_{v}\right)$ (subpixel ellipse center position, the parameters we are interested in), $\lambda_{1}, \lambda_{2}$ the elongation factors of major and minor axes, and $\theta$ the angle between the major axis and $x$ axis.

The intensity model assumes constant intensity in the disk center and in the periphery with a linear transition between both. For luminance level $L_{1}$ at the disk center and $L_{2}$ at its periphery $\left(L_{1}<L_{2}\right)$, the luminance transition is represented by three line segments as in Figure 3, the gradient part being linear with slope $k$. The distances $-\frac{1}{k}$ and $\frac{1}{k}$ define the border of the disk, gradient, and periphery areas.

At a model point $(x, y)$ of (10), the corresponding image point $\left(x^{\prime}, y^{\prime}\right)$ has luminance level $L\left(x^{\prime}, y^{\prime}\right)$ according to Figure 3. We use the Levenberg-Marquardt algorithm to minimize the sum of squared differences of the gray levels between each pixel $\left(x^{\prime}, y^{\prime}\right)$ of the elliptic patch in the image $I$ and point $(x, y)$ of the theoretical CCD model $L$, with respect to the 8 parameters $\left\{\lambda_{1}, \lambda_{2}, \theta, t_{u}, t_{v}, k\right.$, $\left.L_{1}, L_{2}\right\}$ :

$$
\arg \min _{\lambda_{1}, \lambda_{2}, \theta, t_{u}, t_{v}, k, L_{1}, L_{2}} \sum_{x^{\prime}, y^{\prime}}\left(I\left(x^{\prime}, y^{\prime}\right)-L(x, y)\right)^{2} .
$$




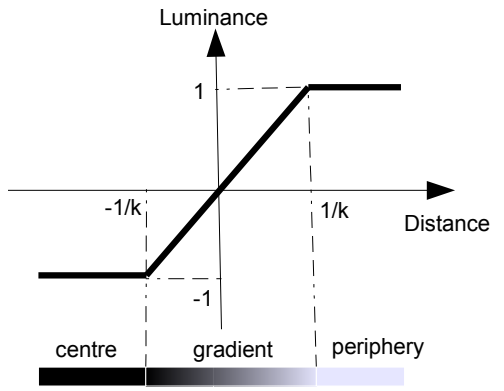

Fig. 3. The luminance transition model for one disk, of parameter $k$

Only the resulting subpixel center coordinates $\left(t_{u}, t_{v}\right)$ are used for color channel alignment.

The process is the following: in each color channel, R, G and B, a simple thresholding gives rough disk locations. Each one is refined through the above minimization and keypoints of $R$ and $B$ channels are paired to nearest keypoint in $G$ channel. This provides a discrete alignment at keypoints of these two channels to the green one. We use the green channel as reference as it is the most represented in the standard Bayer pattern.

\section{Correction Model}

Lateral CA can be considered as geometric distortion between color channels. Assuming the distortion field is smooth by nature, the main idea is to model each distortion field, one for red and one for blue channels, by bivariate polynomials in $x$ and $y$, while keeping one channel as a reference (the green one). The polynomial model, when compared to many other distortion models (radial, division, rational functions), is general and can perfectly approximate the former models [10]. To achieve high precision the degree 11 was chosen for both $x$ and $y$. Experimentally the error stabilizes for polynomials of degrees 7 to 11 [10].

The keypoints are represented in pixel coordinates as $\left(x_{f}, y_{f}\right)$ for a certain color plane $f$, red $(r)$, blue $(b)$ or green $(g)$. The lateral misalignments between the red (or blue) and the green planes are corrected by identifying the parameters of polynomials $p_{f x}, p_{f y}$ approximating at best the equations

$$
\begin{aligned}
& x_{g i}=p_{f x}\left(x_{f i}, y_{f i}\right) \\
& y_{g i}=p_{f y}\left(x_{f i}, y_{f i}\right),
\end{aligned}
$$

with the target colors $f=r$ or $f=b$ and $i$ describing keypoint index. 
The polynomial model $p_{f x}$ of degree $m$ and polynomial coefficients $\left\{p_{x_{0}}\right.$, $\left.p_{x_{1}}, \cdots, p_{x_{\frac{(m+1)(m+2)}{2}-1}}\right\}$ can be expanded as:

$$
\begin{aligned}
x_{g} & =p_{x_{0}} x_{f}^{m}+p_{x_{1}} x_{f}^{m-1} y_{f}+p_{x_{2}} x_{f}^{m-2} y_{f}^{2}+p_{x_{m}} y_{f}^{m}+p_{x_{m+1}} x_{f}^{m-1}+\ldots \\
& +p_{x_{m+2}} x_{f}^{m-2} y_{f}+p_{x_{2 m}} y_{f}^{m-1}+p_{x_{\frac{(m+1)(m+2)}{-3}}} x_{f}+\ldots \\
& +p_{x_{\frac{(m+1)(m+2)}{2}-2}} y_{f}+p_{x} \frac{(m+1)(m+2)}{2}-1
\end{aligned}
$$

The unknowns are the parameters of polynomials $p_{f x}$ and $p_{f y}$. These are $(m+1)(m+2) / 2=78$ for degree $m=11$ for each polynomial. Our pattern is composed of about 1000 disks (we show below that we can pack so many disks of small diameter on our pattern without deterioration of precision), and it also means there is no risk of overfitting because the number of control points is far higher than the number of unknowns. For a number $K$ of keypoints $\left(x_{f i}, y_{f i}\right), i=1, \cdots, K$ distributed all over the image, the polynomial coefficients are computed by minimizing the difference of displacements between the reference and distorted channels:

$$
E=\sum_{i=1}^{K}\left(p_{f x}\left(x_{f i}, y_{f i}\right)-x_{g i}\right)^{2}+\left(p_{f y}\left(x_{f i}, y_{f i}\right)-y_{g i}\right)^{2}
$$

The solution vector $p$ of this least square problem satisfies a linear system $A p=v$ with $A$ the coefficient matrix built from the $x_{f i}, y_{f i}$. For favorable numerical conditioning of $A$, these pixel coordinates need to be normalized between 0 and 1 by application of a global scale.

After the calibration is done, it is straightforward to perform the correction for any image which was taken under the same fixed camera settings. The polynomials $p_{f x}$ and $p_{f y}$ calculate the corrected pixel coordinates for each distorted pixel coordinate $\left(x_{f}, y_{f}\right)$ as in (3), and then the color is obtained by interpolation from the corrected coordinates.

\section{Experiments}

A set of experiments are performed to measure the ellipse center detection accuracy at different ellipse sizes against noise and aliasing. To evaluate the CA correction method we use two types of metrics: geometry and color. Comparison to existing commercial software is done.

\subsection{Keypoint Detection Accuracy}

Synthetic disk (filled circle) 8-bit images were generated on a large image, blurred, downsampled with scale $s=20$ and finally Gaussian noise were added. Subpixel disk center location is used as ground truth and compared to detected disk center. Figure 4 shows the performance of the algorithm against Gaussian 


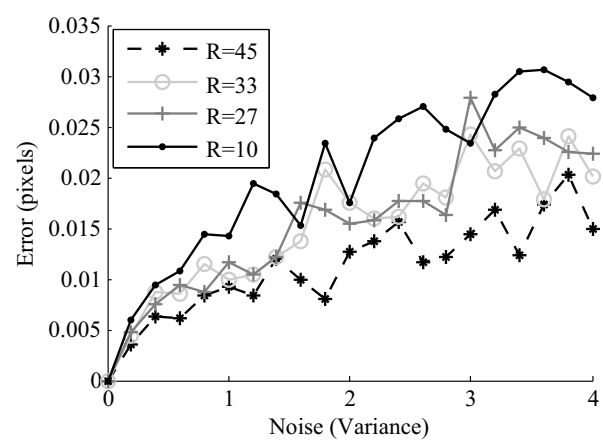

(a) Added Gaussian noise in final image

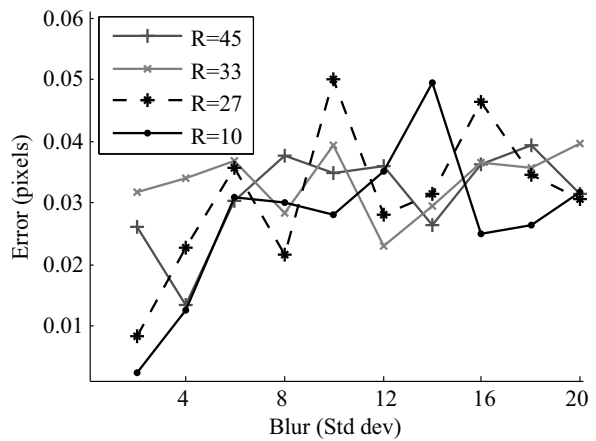

(b) Gaussian blur before downsampling

Fig. 4. Keypoint detection precision for varying disk radii, $R$ (pixels) and downsampled rate $s=20$

noise level added to the images (median error out of 25 iterations) and amount of blur for different disk radii.

It can be observed that in all cases the error is less than 0.05 pixel, even for small disk radius (10 pixels). That allows us to pack more keypoints in a given pattern area. As expected, the error increases roughly linearly with noise level. However, it remains constant under blur. This is important because in a Bayer pattern image, red and blue channels are notoriously aliased. Figure 4 (b) shows that this does not affect the disk centers detection.

\subsection{Geometry Misalignment}

The calibration pattern is printed on an $A 3$ format paper. It is composed of $37 \times 26=962$ black disks of radius $0.4 \mathrm{~cm}$ and separation of $1.1 \mathrm{~cm}$ between consecutive disks. Three digital reflex cameras with interchangeable lenses are used to capture the images: Canon EOS 5D, Canon EOS 40D and Sony DSLR A200. They are noted hereafter 'cam 1', 'cam 2' and 'cam 3'. RAW images are separated in their three channels. The green channel is kept at original resolution of the raw image by bilinear interpolation of missing pixels in the Bayer pattern. Red and blue channels are in (aliased) half-dimension images.

The color planes keypoint displacements are presented in Table 1. Significant reduction is observed after correction. It can be noted that the residuals are of similar magnitude as the keypoint detection, which emphasizes the importance of having precise keypoints.

Further details on keypoint displacements are presented for one case (cam 1 with $f_{1}=24 \mathrm{~mm}$, blue channel) in Figure 5. From the histograms it can be seen that error distribution decreases and stays within 0.05 pixel; this numerical result holds for most of our tests. The vector fields show how the character of the displacement field had changed: before correction it has a fairly radial nature, after correction it is less structured. 
Table 1. Keypoint displacements between color planes in the format "RMSE (maximum)" distances in pixels before and after correction at two focal lengths for each camera

\begin{tabular}{|c|cc||cc|}
\hline & \multicolumn{2}{|c||}{ Uncorrected } & \multicolumn{2}{c|}{ Corrected } \\
Cameras & Red/Green & Blue/Green & Red/Green & Blue/Green \\
\hline cam 1 & & & & \\
$f_{1}=24 \mathrm{~mm}$ & $0.191(0.763)$ & $1.606(3.615)$ & $0.029(0.088)$ & $0.025(0.131)$ \\
$f_{2}=70 \mathrm{~mm}$ & $0.488(0.835)$ & $1.347(1.584)$ & $0.032(0.113)$ & $0.040(0.134)$ \\
\hline cam 2 & & & & \\
$f_{1}=18 \mathrm{~mm}$ & $0.654(0.978)$ & $1.419(3.358)$ & $0.029(0.113)$ & $0.058(0.153)$ \\
$f_{2}=55 \mathrm{~mm}$ & $0.459(0.879)$ & $1.524(2.496)$ & $0.044(0.123)$ & $0.039(0.092)$ \\
\hline cam 3 & & & & \\
$f_{1}=18 \mathrm{~mm}$ & $0.9106(1.1422)$ & $1.5371(3.4125)$ & $0.0344(0.1037)$ & $0.0373(0.0882)$ \\
$f_{2}=70 \mathrm{~mm}$ & $0.2502(0.5382)$ & $1.7066(2.4355)$ & $0.0492(0.1249)$ & $0.0429(0.1502)$ \\
\hline
\end{tabular}
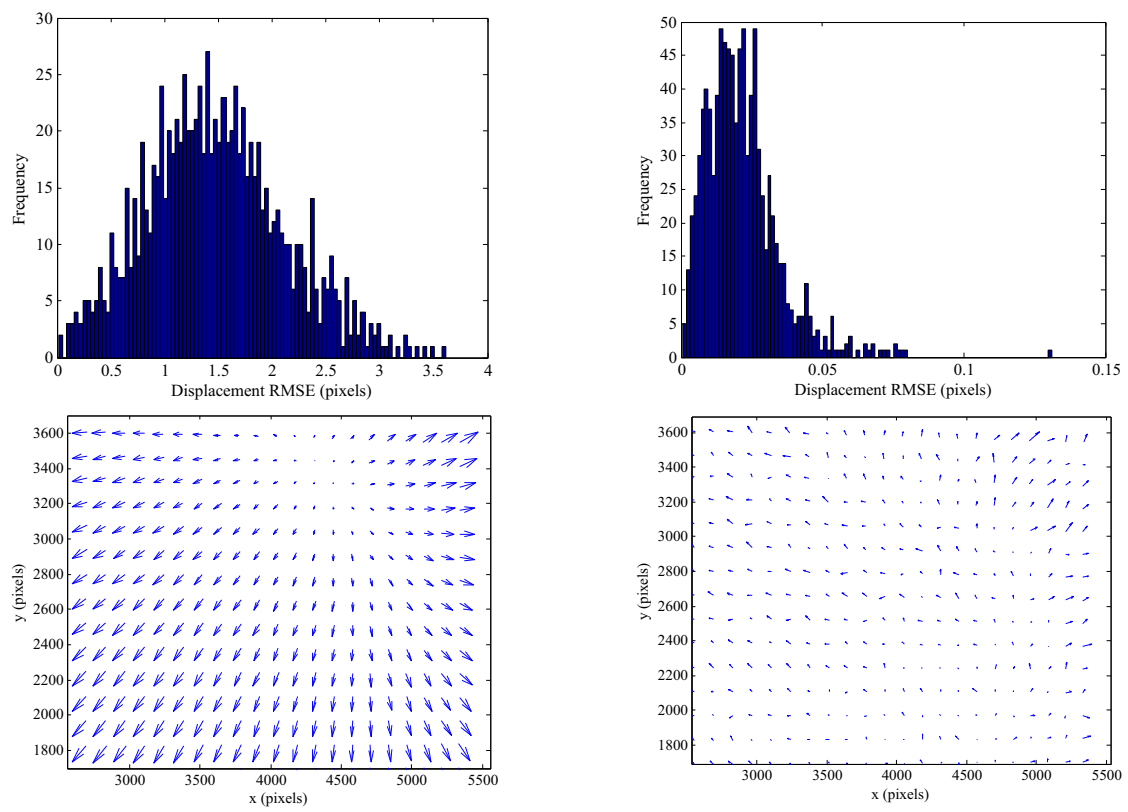

Fig. 5. Histograms (top row) and vector fields (bottom row) of Euclidean displacements of keypoints of the calibration image for blue channel with respect to green channel taken by cam 1 with $f_{1}=24 \mathrm{~mm}$ before (left column) and after (right column) correction 
Three software solutions were chosen to perform a comparison with our method: DxO Optics Pro (noted as 'DxO') 11, Canon DPP ('DPP') 12] and Jaliko lenstool ('JLT') [13. The first two use lab-computed calibration database for each camera and each lens, and perform correction based on the database, with possibility of manual readjustment for each image. The CA correction by JLT method is fully automatic and for any kind of camera (no database) but requires several images. Cam 2 was chosen for this experiment and the keypoint displacement results are shown in Figure 6 for different focal lengths. The results demonstrate that only our method achieves precision where defects are not visible anymore (see Figure 2): the mean always stays within $0.05-0.1$ pixel while for other methods the average remains around 0.4 pixels.

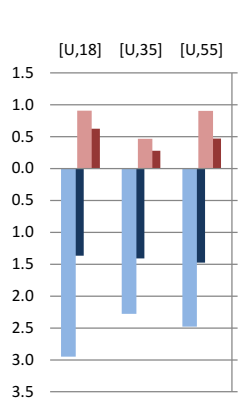

(a) Uncorrected

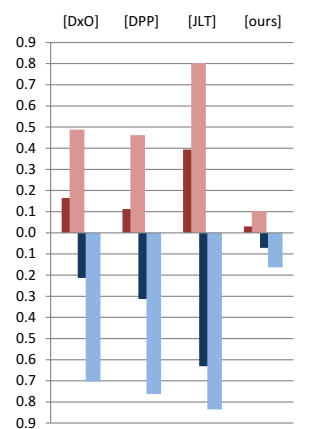

(b) $f=18 \mathrm{~mm}$

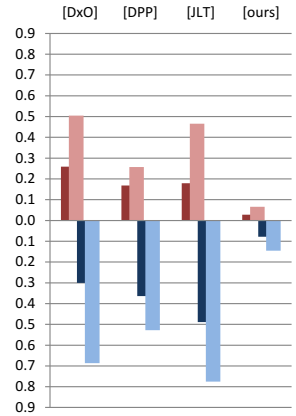

(c) $f=35 \mathrm{~mm}$

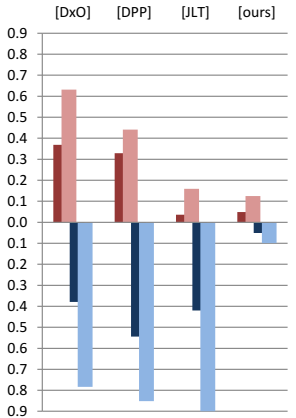

(d) $f=55 \mathrm{~mm}$

Fig. 6. Comparison of our method to other software for cam 2 and different focal lengths $f$, the comparison is in terms of mean (dark colored bars) and maximum (light colored bars) misalignments for both red (positive axis) and blue (negative axis) channels. (a) provides information on the initial uncorrected keypoint displacements for the same focal lengths (notice the different scale).

\subsection{CA Correction: Color Misalignment}

To visualize the effect of CA correction, color based $3 D$ point cloud is built for the calibration image. Since we use a black and white pattern, it is expected that $3 D$ color cloud of the calibration image would lie along the gray line connecting black and white spots. If chromatic aberration is present, color channels are not aligned and occurring blue-red hues come out at significant distance from the gray line, thus creating a bulged $3 D$ color cloud. Figure 7 shows two color clouds built in RGB space before and after the correction for cam 1 at $f_{1}=24 \mathrm{~mm}$.

To get a quantitative evaluation of the color cloud, we use statistics on the distances $d_{x}$ from each color point to a local gray line. To compensate the absence of white balance in the RAW image, we use the local gray line, representing $3 D$ regression of the point cloud (obtained by eigen decomposition of the variancecovariance matrix of the color cloud). Figure 8 shows the frequency histogram of 

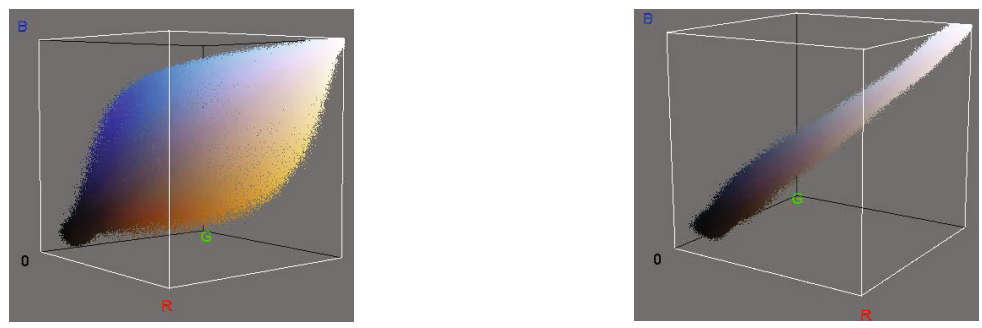

Fig. 7. $3 D$ color clouds of the calibration image before (left) and after (right) correction

color pixels in the calibration image. Obviously, the majority of pixels belong to the regions of the local black and white spots and these have naturally almost vanishing error. To get more significant statistics, we eliminate all those majority pixels. The distances of the remaining points to the local gray line are used to get a quantitative measure:

$$
S=\sqrt{\frac{\sum_{x} d_{x}^{2}}{N}}
$$

Assuming the gray line passes through two points $X_{1}^{(g)}, X_{2}^{(g)}$, the distance from

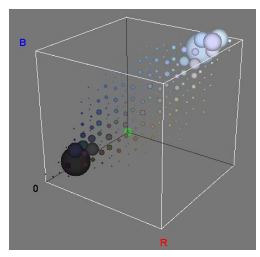

Fig. 8. Frequency histogram representation

a color point $x$ to the gray line is expressed as: $d_{x}=\frac{\left|\left(x-X_{1}^{(g)}\right)\left(x-X_{2}^{(g)}\right) e_{3}\right|}{\left\|X_{2}^{(g)}-X_{1}^{(g)}\right\|}$, with the numerator being a determinant of a $3 \times 3$ matrix whose third column is the vector $e_{3}=\left(\begin{array}{lll}0 & 0 & 1\end{array}\right)^{T}$. Table 2 shows the RMSE and maximum distances for the calibration image and two cameras. The color misalignment can be reduced up to 6 times, depending on the camera. The plot of color error around two disks of the pattern image for cam 1 is shown in Figure 9 as gray level values (white is maximum, black is zero). After correction, the error magnitude for chromatic aberration is comparable with noise level, as opposed to the plot before correction, where noise is masked by the amplitude of color error.

\subsection{Visual Improvement for Real Scenes}

To see the improvement in image quality, a zoomed-in crops of outdoor images are shown Figure 10 and Figure 11, Red and blue fringes can be seen in original images. They disappear after correction. The same effect can be observed in Figure 1 
Table 2. Error measures of color distribution in RMSE (maximum distance) format

\begin{tabular}{|c|c|c|c|}
\hline Camera & $\begin{array}{c}\text { Uncorrected, } \\
S_{u}\left(d_{u, \max }\right)\end{array}$ & $\begin{array}{l}\text { Corrected, } \\
S_{c}\left(d_{c, \max }\right)\end{array}$ & $\begin{array}{c}\text { Ratio, } \\
\frac{S_{u}}{S_{c}}\left(\frac{d_{u, \max }}{d_{c, \max }}\right)\end{array}$ \\
\hline cam 1 & & & \\
\hline$f_{1}=24 \mathrm{~mm}$ & $41.54(78.21)$ & $6.91(22.87)$ & $6.01(3.41)$ \\
\hline$f_{2}=70 \mathrm{~mm}$ & $30.74(68.98)$ & $5.92(18.33)$ & $5.19(3.76)$ \\
\hline cam 2 & & & \\
\hline$f_{1}=18 \mathrm{~mm}$ & $32.35(66.07)$ & $9.46(34.08)$ & 3.41 (1.93) \\
\hline$f_{2}=55 \mathrm{~mm}$ & $36.48(78.73)$ & $7.35(31.32)$ & $4.96(2.51)$ \\
\hline cam 3 & & & \\
\hline$f_{1}=18 \mathrm{~mm}$ & $19.99(50.18)$ & $5.75(24.45)$ & $3.47(2.05)$ \\
\hline$f_{2}=70 \mathrm{~mm}$ & $28.51(94.54)$ & $7.22(76.76)$ & $3.94(1.23)$ \\
\hline
\end{tabular}
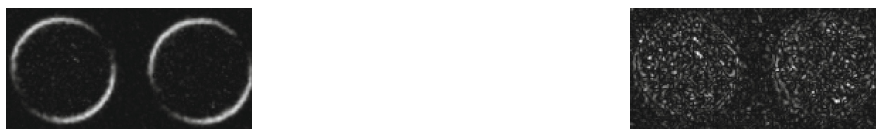

Fig. 9. Error distances (5) to the local gray line for the calibration image of cam 1 , before (left) and after (right) correction
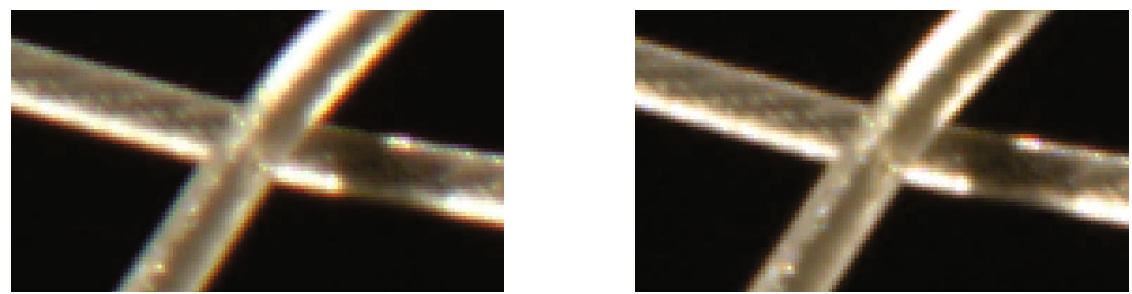

Fig. 10. Cropped and zoomed-in image from camera 1, before (left) and after (right) correction
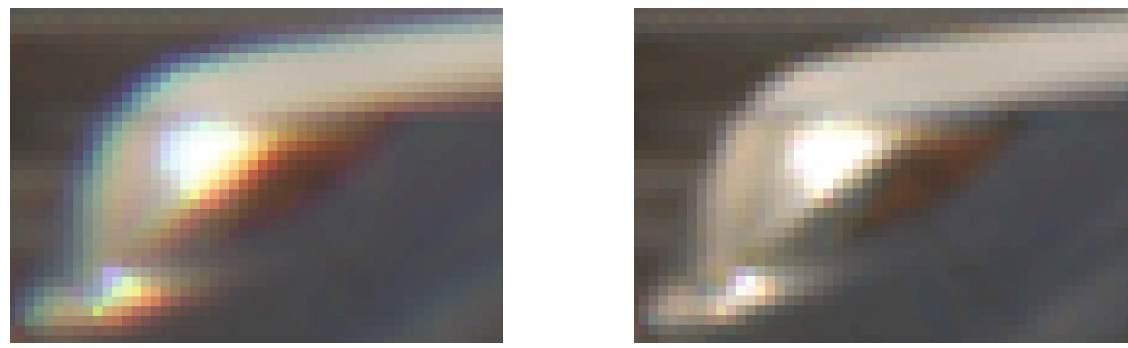

Fig. 11. Cropped and zoomed-in image from camera 3, before (left) and after (right) correction 


\section{Conclusion}

We have proposed a high precision chromatic aberration correction method, using a single snapshot of a pattern of black disks. Their centers are used as keypoints and aligned between different channels. A dense correction vector field is then deduced by a general polynomial model. Though already quite accurate, the precision is limited by the keypoint location detection. Comparison tests showed that our method outperforms existing software solutions by a significant margin. Further work includes comparison with a pattern made of pure noise, producing numerous SIFT [14] keypoints. Whereas their precision is likely to be lower, their number could compensate that and may improve the overall correction.

Acknowledgments. This work was carried out in IMAGINE, a joint research project between École des Ponts ParisTech (ENPC) and the Scientific and Technical Centre for Building (CSTB). Part of this work was funded by the Agence Nationale de la Recherche, Callisto project (ANR-09-CORD-003).

\section{References}

1. Farrar, N.R., Smith, A.H., Busath, D.R., Taitano, D.: In-situ measurement of lens aberrations. In: Optical Microlithography, vol. XIII (2000)

2. Millán, M.S., Otón, J., Pérez-Cabré, E.: Dynamic compensation of chromatic aberration in a programmable diffractive lens. Optics Express 14, 9103-9112 (2006)

3. Willson, R., Shafer, S.: Active lens control for high precision computer imaging. In: Proc. IEEE ICRA, vol. 3, pp. 2063-2070 (April 1991)

4. Kozubek, M., Matula, P.: An efficient algorithm for measurement and correction of chromatic aberrations in fluorescence microscopy. Journal of Microscopy 200(3), 206-217 (2000)

5. Boult, T.E., Wolberg, G.: Correcting chromatic aberrations using image warping. In: 1992 IEEE Computer Society Conference on Computer Vision and Pattern Recognition, Proceedings CVPR 1992, pp. 684-687. IEEE (1992)

6. Matsuoka, R., Asonuma, K., Takahashi, G., Danjo, T., Hirana, K.: Evaluation of correction methods of chromatic aberration in digital camera images. In: ISPRS Photogrammetric Image Analysis, vol. I-3 (2012)

7. Kaufmann, V., Ladstdter, R.: Elimination of color fringes in digital photographs caused by lateral chromatic aberration. In: CIPA XX International Symposium (2005)

8. Luhmann, T., Hastedt, H., Tecklenburg, W.: Modelling of chromatic aberration for high precision photogrammetry. In: Proc. of the ISPRS Commission V Symp. on Image Engineering and Vision Metrology, vol. 36, pp. 173-178 (2006)

9. Mallon, J., Whelan, P.F.: Calibration and removal of lateral chromatic aberration in images. Pattern Recognition Letters 28(1), 125-135 (2007)

10. Tang, Z.: High accuracy measurement in 3D stereo reconstruction. PhD thesis, ENS Cachan, France (2011)

11. DxO: DxO Optics Pro, http://www.dxo.com/intl/photography/dxo-optics-pro

12. Canon: Digital Photo Professional, http://www.canon.com/

13. Dunne, A., Mallon, J.: Jaliko LensTool (2010), http://www.jaliko.com/

14. Lowe, D.G.: Distinctive image features from scale-invariant keypoints. Int. J. Comput. Vision 60(2), 91-110 (2004) 